\title{
The Laboratory Diagnosis and Clinical Management of Patients with Heparin- Induced Thrombocytopenia: An Update
}

\author{
Margaret Prechel, Ph.D., ${ }^{1}$ and Jeanine M. Walenga, Ph.D. ${ }^{2}$
}

ABSTRACT

Heparin-induced thrombocytopenia (HIT) is a serious adverse effect of heparin exposure that can progress to severe thrombosis, amputation, or death. HIT is an immune response in which antibodies cause platelet activation, platelet aggregation, the generation of procoagulant platelet microparticles, and activation of leukocytes and endothelial cells. Early diagnosis based on a comprehensive interpretation of clinical and laboratory information is important to improve clinical outcomes. However, limitations of the laboratory assays and atypical clinical presentations can make the diagnosis difficult. Clinical management of patients with HIT is with a non-heparin anticoagulant such as a direct thrombin inhibitor or danaparoid followed by a vitamin $\mathrm{K}$ antagonist for long-term treatment. The new anti-factor Xa drugs (fondaparinux, rivaroxaban, apixaban) and other non-heparin antithrombotic agents can potentially be used for the treatment of HIT if clinically validated. Important drug-specific limitations and dosing and monitoring guidelines must be respected for patient safety. Issues still exist regarding the optimal clinical management of HIT.

KEYWORDS: Heparin, thrombocytopenia, antibody, assays, thrombin inhibitor

The clinical effects of heparin are meritorious, and heparin remains the anticoagulant of choice for most clinical needs. However, as with any drug, adverse effects exist. Heparin-induced thrombocytopenia (HIT) is an important adverse effect of heparin. Because heparin is used ubiquitously in the hospital setting, millions of patients are exposed each year. It is important to be aware of and understand HIT because of the devastating clinical consequences of amputation and death due to thrombosis with which it is commonly associated. Although the diagnosis and treatment of HIT can be difficult and complex, it is critical that patients with HIT be identified as soon as possible to initiate early treatment to avoid thrombosis.

\section{PATHOPHYSIOLOGY OF HIT}

HIT is an immune-mediated adverse response to heparin treatment. ${ }^{1-3}$ HIT antibodies are not directed toward heparin specifically but rather to platelet factor 4 (PF4) in complex with heparin. PF4 is a positively charged protein stored in the $\alpha$ granules of platelets. Exposed lysine and arginine residues on the tetrameric PF4 molecule bind to negatively charged heparin
${ }^{1}$ Assistant Professor, Department of Pathology, Stritch School of Medicine, Loyola University Chicago, Maywood, Illinois; ${ }^{2}$ Professor, Departments of Thoracic \& Cardiovascular Surgery and Pathology, Stritch School of Medicine, Loyola University Chicago, Maywood, Illinois.

Address for correspondence and reprint requests: Dr. Jeanine M. Walenga, Cardiovascular Institute, Building 110, Room 5226, Loyola lumc.edu).

Hot Topics II: An Editorial Collection of Current Issues and Controversies in Thrombosis and Hemostasis; Guest Editor, Emmanuel J. Favaloro, Ph.D., M.A.I.M.S.

Semin Thromb Hemost 2008;34:86-96. Copyright (C) 2008 by Thieme Medical Publishers, Inc., 333 Seventh Avenue, New York, NY 10001, USA. Tel: +1(212) 584-4662.

DOI 10.1055/s-2008-1066027. ISSN 0094-6176. 
molecules. ${ }^{4,5}$ This binding exposes cryptic regions within the PF4 molecule creating antigenic neoepitopes. ${ }^{6-8}$ Multiple PF4 tetramers arrayed in a lattice with several molecules of heparin are highly immunogenic and play a fundamental role in antibody formation. ${ }^{9}$ Antibodies formed in response to the heparin:PF4 complex (H:PF4) subsequently recognize PF4 bound to cell membranes ${ }^{10}$ or other surfaces. ${ }^{11}$ Antibodies to other heparin-binding proteins, such as neutrophil activating peptise 2 (NAP-2) and IL-8, have been identified; however, those to $\mathrm{PF} 4$ are found in most patients with HIT. $^{12,13}$

Because of its smaller molecular size, low-molecular-weight heparin (LMWH) has less ability to bind to the PF4 tetramer, alter its configuration, and cause the generation of HIT antibodies. Patients treated with LMWH are 2 to 3 times less likely to develop HIT antibodies than are patients treated with unfractionated heparin (UFH). However, in vitro studies demonstrate that LMWH cross-reacts with existing HIT antibodies formed in response to UFH. ${ }^{14}$

An interesting development has recently occurred with the introduction of generic LMWHs. Regulatory bodies are challenged to develop specific guidelines for generic LMWH approval due to the complex nature of these polycomponent biologicals. The U.S. Food and Drug Administration (FDA) has identified immunogenicity of LMWHs as an important criterion to differentiate LMWHs and to demonstrate the bioequivalence of generic LMWHs to the branded products. ${ }^{15}$

The immunogenic potential of heparins is more complex than that of protein-derived drugs such as hirudin, aprotinin, factor VIII concentrate, and erythropoietin. Because of their polycomponent nature and their multiple interactions with endogenous proteins such as PF4, fibronectin, histidine-rich glycoprotein, growth factors, and serpins, heparins and related polysaccharides likely generate an array of antibodies some of which may modulate their pharmacologic actions. Therefore, apart from HIT antibodies and their associated pathology, patients generating heparin-mediated antibodies may exhibit a therapeutic compromise requiring dose adjustment of the heparin or an alternative approach for anticoagulation.

HIT antibodies, once formed, become involved in various hemostatic activation processes. Immune complexes of HIT IgG and H:PF4 cross-link platelet Fc $\gamma I I a$ receptors, resulting in platelet activation and release of additional PF4. In the presence of heparin, there is continued formation of antigenic complexes, initiating a cycle of platelet activation and aggregation and generation of highly procoagulant platelet microparticles. ${ }^{1,6,16,17}$ Sustained platelet activation contributes to platelet clearance and thrombin generation that can lead to both thrombocytopenia and HIT-associated thrombosis.
Platelets activated by HIT antibodies induce an inflammatory state in which macrophages, monocytes, and neutrophils are activated. ${ }^{16,18-20}$ Antibody and leukocyte binding to activated endothelial cells causes release of tissue factor, plasminogen activator inhibitor-1 (PAI-1), and cytokines, as well as an upregulation of adhesion molecule expression promoting localized platelet and monocyte binding. ${ }^{18,21-24}$ Heparan sulfate on the endothelial cell surface can bind PF4, forming a complex that is recognized by HIT antibodies. ${ }^{10,24}$ The interrelationships of platelets, leukocytes, the endothelium, and the inflammatory state determine the clinical expression of HIT.

\section{FREQUENCY OF HIT}

$\mathrm{H}: \mathrm{PF} 4$ antibodies are necessary but not sufficient to cause the clinical symptoms of HIT (thrombocytopenia and thrombosis) as many patients who develop HIT antibodies remain asymptomatic. Heparin derived from bovine sources is more immunogenic than is porcine heparin, and UFH elicits more antibody formation than does LMWH. Risk of HIT increases with longer treatment duration and is more likely with intravenous heparin than with subcutaneous administration. Still, HIT can develop from any heparin exposure, including incidental amounts from heparin flushes or heparincoated devices.

Frequency of seroconversion and development of thrombocytopenia and/or thrombosis associated with HIT are variable and depend on factors such as patient population and presence of comorbid complications. ${ }^{25-27}$ HIT antibodies are more likely to form in surgical patients than in medical patients, and sicker patients (e.g., patients with malignancy, sepsis, or vascular disease) and older patients are at higher risk.

Development of HIT-induced thrombocytopenia with or without thrombosis is not always proportional to the risk of seroconversion. Whereas 25 to $50 \%$ of cardiac surgery patients form antibodies, less than $2 \%$ develop the clinical symptoms of HIT. Among orthopedic patients, $15 \%$ can be antibody positive but only $5 \%$ develop clinical consequences. ${ }^{28}$ Overall, clinically symptomatic HIT develops in $1 \%$ of hospital patients receiving heparin in any form. ${ }^{29}$

\section{CLINICAL PRESENTATION OF HIT}

HIT is typically described as an otherwise unexplained thrombocytopenia starting 4 to 14 days after administration of heparin. Thrombocytopenia is usually defined as a platelet count $<100 \times 10^{9} / \mathrm{L}$ to $150,000 \times 10^{9} / \mathrm{L}$; however, HIT may also be recognized by a 30 to $50 \%$ drop from the preheparin baseline even if the platelet count remains higher than this range. No single definition of thrombocytopenia is appropriate in all clinical 
situations. ${ }^{30}$ HIT is particularly difficult to diagnose in patient populations where low platelet counts are typical. In orthopedic and cardiac surgery patients, HIT may be recognized by the pattern of platelet count recovery or by a particular percent decrease compared with the postsurgical platelet level. Patients requiring ventricularassist devices who receive anticoagulation during surgery and for extended postoperative periods of mechanical circulatory support often develop H:PF4 antibodies, but they also have multiple explanations for low platelet counts. ${ }^{31}$ No guidelines are yet established for HIT in this patient group.

In a heparin naive patient, typical onset of HIT is delayed several days, representing the time course of $\mathrm{H}: \mathrm{PF} 4$ antibody generation. In a patient with previous heparin exposure, particularly within the most recent 120 days, H:PF4 antibodies may already be present and lead to early onset of HIT, even within hours of the next heparin exposure. Currently, many patients are discharged from hospitals within several days after exposure to heparin, and in these cases a symptomatic late drop in platelet count goes undetected. In the event that these patients suffer thrombotic complications and return to the hospital, failure to rule out the possibility of HIT prior to administering therapeutic heparin can lead to catastrophic consequences.

Despite the hallmark low platelet count, HIT patients rarely have bleeding complications. The major significance of HIT is the paradoxical risk of thrombosis, which occurs in $30 \%$ of HIT patients with thrombocytopenia. In some patients, thrombosis is apparent at the time of HIT diagnosis; patients initially without thrombosis have up to $50 \%$ risk of developing this complication within the next 30 days if not treated with a non-heparin anticoagulant. There is a wide spectrum of arterial and venous thromboembolic complications associated with HIT, including deep vein thrombosis, pulmonary embolism, myocardial infarction, thrombotic stroke, ischemic limb, vein graft occlusion, and skin lesions at injection sites. Mortality among patients with HIT thrombosis is up to $30 \%$, with $20 \%$ of those surviving requiring a limb amputation.

Diagnosis of HIT is based primarily on clinical presentation. Diagnostic scoring systems have been proposed to help clinicians evaluate clinical impressions based on the timing and extent of thrombocytopenia and the presence or absence of thrombotic complications or other explanations for low platelet count. ${ }^{32,33}$ The performance of such risk assessment strategies varies with the experience of the clinician. ${ }^{34}$

\section{LABORATORY DIAGNOSIS OF HIT}

There are two types of laboratory tests for HIT. Antigen assays detect the presence of immunoglobulins that bind the antigenic neoepitopes exposed in $\mathrm{H}: \mathrm{PF} 4$ complexes.
Functional tests detect the presence of HIT antibody immune complexes that cause platelet activation. Each type of test provides unique and complementary information. None of the laboratory tests should be used as the sole basis to rule-in or rule-out the diagnosis of HIT.

\section{Platelet Function Tests}

The functional tests are bioassays that use fresh platelets from a known reactive normal donor incubated with patient's serum or plasma. Addition of an appropriate concentration of heparin to the PF4 that is present in the platelet/specimen incubation allows $\mathrm{H}: \mathrm{PF} 4$ complexes to form and present the HIT antigen. HIT antibodies bound to the H:PF4 in immune complexes cause platelet activation. Different functional tests use specific platelet activation end points.

The serotonin release assay (SRA) is conducted with platelets that have been incubated with ${ }^{14} \mathrm{C}$ radiolabeled serotonin then washed and resuspended in calcium-containing assay buffer. Platelets are Fcy receptor-bearing cells that are activated by $\operatorname{IgG}$ immune complexes. Platelet activation resulting in granule release is detected by the presence of radioactivity in the incubation supernatant. By measuring background radioactivity and total ${ }^{14} \mathrm{C}$ uptake, the strength of the activation response to HIT IgG can be quantified as percent serotonin release. Platelet activation is usually defined as $20 \%$ or greater serotonin release. Stronger release activity presumably indicates high affinity or high titer antibody; 50 to $80 \%$ release has been shown to be more specific for HIT patients with thrombocytopenia and/or thrombosis. ${ }^{32}$ An important control in platelet activation assays used for diagnosis of HIT is inclusion of both low $(0.1 \mathrm{U} / \mathrm{mL})$ and high $(100 \mathrm{U} / \mathrm{mL})$ heparin incubations with each specimen. A positive result in a HIT diagnostic assay is platelet activation in the presence of low heparin but not in the presence of high heparin. An activation assay result is "indeterminate" when a specimen causes platelet activation at both low and high concentrations of heparin, which indicates that the antigenic target is not heparindependent. These specimens may contain preformed immune complexes or antibodies such as anti-HLA or antiplatelet glycoprotein antibodies. The two-point assay design for HIT diagnosis increases the specificity of the in vitro assays by minimizing "false-positive" results.

A similar washed platelet activation assay is the heparin induced platelet aggregation (HIPA) assay, which uses a visual assessment of platelet aggregation over time. The strength of the activation response in this assay is reflected in the lag time until aggregation is observed. HIT antibody immune complexes can also be detected by ADP release measured by lumi-aggregometry or platelet microparticle formation or annexin binding detected by flow cytometry. 
Other functional tests conducted with patient serum or plasma, heparin, and donor platelets use citrated plasma. The platelet aggregation test (PAT) is conducted in a commercial aggregometer, which measures platelet activation by the percent increase in light transmission through the platelet suspension that occurs as platelets aggregate. Tests conducted in citrated platelet-rich plasma are considered less sensitive than washed platelet assays; however, patient specimens can test positive by PAT but negative by SRA and vice versa. ${ }^{33,35}$

There is considerable donor-related variability in platelet responsiveness in these functional platelet assays, making it imperative that tests are performed with platelets from known reactive donors. ${ }^{36,37}$ Each assay should also include known HIT antibody-positive and antibody-negative control sera. The functional tests for HIT are highly complex, difficult to standardize, and require careful attention to quality control measures. The most reproducible results are obtained when these assays are conducted in experienced reference laboratories. ${ }^{38}$

\section{Antigen Assays}

Antigen assays for HIT detect antibodies that recognize and bind cryptic PF4 epitopes. Two solid-phase enzyme-linked immunosorbent assays (ELISAs) are commercially available. The Asserachrom ELISA (Diagnostica Stago, Parsippany, NJ) provides assay wells coated with heparin in complex with recombinant human PF4; the GTI HAT ELISA (Genetics Testing Institute, Waukesha, WI) uses negatively charged polyvinylsulfonate binding to expose antigenic epitopes in PF4 isolated and purified from human platelets. Both assays use enzyme-labeled anti-human immunoglobulin conjugates and a suitable substrate to produce color in wells with bound HIT antibodies. Results are expressed as optical density (OD); cutoff OD values are provided by the manufacturers. HIT antibodies are polyclonal and differ in specificity and affinity. It is of interest that these two ELISA kits detect slightly different cohorts of H:PF4 antibodies, ${ }^{39}$ giving opposite results in $15 \%$ of specimens tested by both methods. ${ }^{40,41}$

Initially, the commercially available ELISA kits used a global (anti-IgG, IgA, IgM) conjugate reagent. Because studies have shown that platelet-activating (SRA testing) HIT antibodies are of the IgG isotype, ${ }^{42,43}$ ELISAs are now available to detect only HIT $\mathrm{IgG}$. Whereas the $\mathrm{IgG}$-specific assay is more sensitive for overt HIT where patients have marked thrombocytopenia and thrombosis, the global assay may provide earlier evidence of a progressing immune reaction.

As with platelet activation tests, addition of high heparin to ELISA wells inhibits HIT antibody binding by removing or blocking access to antigenic PF4 epitopes. ${ }^{44}$ Wells with antibodies that bind nonspecifically to the ELISA plate will have positive OD values in the presence as well as in the absence of high heparin. There is data to indicate that when antibody binding was not inhibited by high heparin, patients were unlikely to have HIT, suggesting that the extra step provides useful diagnostic information. ${ }^{45}$

Two antigen-based rapid detection HIT antibody assays have been developed. The particle gel immunoassay (PaGIA) is an agglutination assay that uses polystyrene beads coated with H:PF4 complexes and test serum/plasma incubated in a chamber of an IDMicroTyping test card (DiaMed, Cressier Sur Morat, Switzerland). Centrifugation of the test card separates the beads cross-linked by H:PF4 complexes, which are identified by visual inspection. A more qualitative assessment can be done by testing serial dilutions of specimen and reporting antibody titer in terms of the highest dilution showing a positive result. The Particle Immunofiltration Assay (PIFA; Akers Biosciences, Thorofare, NJ) uses microparticles coated with PF4 within a selfcontained minireactor device. Addition of (non-frozen/ thawed) serum containing H:PF4 antibodies will cause matrix formation and trap the microparticles within the chamber membrane. Nonmatrixed microparticles, in HIT antibody-negative specimens, migrate through the membrane and are detected in the test result window of the device.

\section{Interpretation of Assay Results}

The SRA is considered to be the gold standard test for HIT antibodies, and antigen assay performance is generally evaluated in terms of agreement with results of the SRA. Many specimens that test positive by ELISAs do not cause platelet activation in the SRA and are not associated with clinically symptomatic HIT (i.e., patients have HIT antibodies, but these antibodies do not cause platelet activation, and the patients do not have thrombocytopenia or thrombosis). The ELISAs then are less specific tests for HIT. ${ }^{42}$ Negative results in antigen tests, including the rapid PaGIA, reliably rule-out HIT in patients with low probability of HIT based on their clinical symptoms. ${ }^{46}$

In the clinical setting, both platelet activation and antigen tests are reported as either positive or negative. Retrospective research studies suggest that information about the magnitude of a positive response could be helpful in making a diagnosis of HIT. Patients with stronger activation results (50 to $80 \%$ serotonin release) and/or higher antibody titers (OD 1.0 to 1.2) have a greater likelihood of having clinically symptomatic HIT. ${ }^{47,48}$ When evaluating weaker assay responses, it is important to remember that results might increase on a repeat, subsequent test. ${ }^{49,50}$

The interpretation and value of HIT diagnostic tests vary with the timing of the collection of the patient 
specimen and the clinical status of the patient. Preoperative testing may be useful in surgical patients with recent heparin exposure or previous history of clinically symptomatic HIT or in patients with inflammatory or malignant complications. Assuming a negative preoperative antibody result, tests within 1 to 4 days after heparin exposure provide little information, as $\mathrm{H}: \mathrm{PF} 4$ antibodies would not yet be apparent.

The most relevant information results from HIT tests done on symptomatic patients (i.e., patients with thrombocytopenia and/or thrombosis) during the period 5 to 14 days after heparin exposure. When adequate assessment of the clinical relevance of platelet count and/ or thrombotic symptoms gives rise to reasonable suspicion of HIT, a positive laboratory result can rule-in the diagnosis and justify initiating or maintaining alternative anticoagulant therapy. When clinical suspicion is high, one negative test should not rule-out HIT. Repeat testing or use of an additional type of test is advisable, along with careful surveillance of platelet counts.

Interpretation of test results, particularly in antigen assays, in patients without clinical symptoms of HIT during the postheparin interval is problematic. Many patients have positive antibody titers and do not develop thrombocytopenia or thrombosis. Other patients may have a negative test but develop symptoms and positive test results on subsequent days (e.g., delayed-onset HIT). Thus, neither test result is informative in terms of patient care.

Until there is more information on risks associated with HIT-seropositivity itself, routine screening of patients without thrombocytopenia or unexplained thrombosis is not recommended.

\section{CLINICAL MANAGEMENT OF HIT PATIENTS}

Current clinical practice for the management of patients with HIT is high clinical awareness, early diagnosis, and early treatment. The diagnosis of HIT is based on a comprehensive interpretation of clinical and laboratory information. HIT is largely a clinical diagnosis (i.e., thrombocytopenia and/or new thromboembolic events). Once HIT is suspected, there is a necessity for immediate intervention to initiate treatment against the high risk of thrombosis. One should not wait for laboratory results to act. It is important, however, to have laboratory confirmation of HIT because patients who have HIT are at great risk for recurrence should they be exposed to heparin in the future. ${ }^{51}$

\section{ANTICOAGULATION OF PATIENTS WITH HIT}

When the diagnosis of HIT is suspected, heparin should be stopped and a substitute anticoagulant introduced immediately. Cessation of heparin alone is not sufficient to remove the threat of thrombosis. ${ }^{52}$ Because individuals with HIT are at extremely high risk for developing thrombosis, and it is not always possible to identify patients with thrombosis, it is advisable to treat all patients prophylactically against thrombosis.

LMWH is contraindicated in patients with HIT because it has a high rate of interacting with established HIT antibodies. Platelet transfusions are also contraindicated in patients with HIT.

Past treatment options for the patient with HIT included dextran, warfarin alone, ancrod, intravenous gamma globulin, prostacyclin, and aspirin. Results were variable and less than optimal. These therapies have become outmoded.

\section{Direct Thrombin Inhibitors}

The success of direct thrombin inhibitors (DTIs) for the management of HIT is due to the difference in chemical structure from heparin such that these drugs do not generate HIT antibodies nor do they interact with preformed HIT antibodies. DTIs are potent anticoagulants that inhibit the high level of thrombin generation in patients with HIT.

Clinical studies have shown that anticoagulation with a DTI significantly reduces the risk of thrombosis and thromboembolic complications (new thrombosis, amputation, death) associated with HIT. ${ }^{53-56}$ The clinical outcomes of all-cause death, amputation, or new thrombosis were improved with treatment. Significantly more treated HIT patients remained event-free compared with controls. Platelet counts recovered more rapidly in patients receiving DTI treatment.

Because of the inherent bleeding risk with all DTIs, it is important to monitor patient treatment. Particular attention should be given to elderly patients and patients with renal/liver failure.

The activated partial thromboplastin time test (aPTT) has been recommended as the monitoring assay for DTIs. Specific values for the aPTT differ by drug and aPTT reagent/instrument system. However, in general, DTIs are dosed to a 2- to 2.5-fold increase in the aPTT. Higher aPTT results are associated with an increased risk of bleeding.

Laboratory tests that use a clotting end point, such as fibrinogen and coagulation factor assays, will be affected by a DTI "contaminant" in the patient's specimen. ${ }^{57,58}$ True factor levels can be measured by chromogenic- or immunologic-based assays that are not affected by the DTI.

Although there are many similarities among the DTIs, important differences exist between them..$^{51,59,60}$ The different chemical structure of each DTI defines where each drug binds to thrombin, the tightness of the binding, and so on. These characteristics are reflected in 
the different pharmacokinetic and pharmacodynamics of each drug.

\section{ARGATROBAN}

Argatroban can be distinguished from other DTIs in that it produces an increase in nitric oxide, which may contribute to its therapeutic efficacy by modulating vascular and cellular function. ${ }^{61}$

Argatroban is hepatically metabolized. Plasma argatroban levels are rapidly reversed in $\sim 40$ minutes when drug is discontinued, and coagulation parameters generally return to pretreatment values within 2 to 4 hours. ${ }^{62-64}$ Argatroban is the anticoagulant of choice in patients who have renal failure. ${ }^{65}$

In the clinical trials for the management of HIT, patients received continuous intravenous argatroban at a dose of 1.7 to $2.0 \mu \mathrm{g} \mathrm{kg}{ }^{-1} \mathrm{~min}^{-1}$ for 5 to 7 days. ${ }^{53,54}$ Similar initial doses were used for prophylaxis and treatment adjusted by the aPTT. Major and minor bleeding rates were similar between treatment and control groups. Analysis of the patients who had a repeat exposure to argatroban revealed no generation of antibodies to argatroban and no bleeding or other adverse events up to 30 days after cessation of therapy. ${ }^{66,67}$ Argatroban has been approved by the health authorities of the United States, Canada, and Europe for both the prophylaxis and treatment of HIT thrombosis.

Argatroban has also been approved by the FDA for anticoagulation of HIT patients during percutaneous coronary interventions (PCI). ${ }^{68}$ There are also reports on the successful use of argatroban anticoagulation for pediatric interventional cardiology procedures and for stent implantation in renal arteries. ${ }^{62,69,70}$ Argatroban anticoagulation in PCI (at a reduced dose) used in combination with glycoprotein IIb/IIIa inhibition for PCI was well tolerated with an acceptable bleeding risk. $^{71}$

In a more recent study, argatroban was found to effectively reduce new stroke and stroke-associated mortality in patients with HIT without increasing intracranial hemorrhage. ${ }^{72}$

\section{LEPIRUDIN}

Lepirudin acts as an irreversible inhibitor with an elimination half-life of $\sim 90$ minutes (1.3 to 3 hours). It is renally excreted and needs to be used with caution in patients with renal impairment.

In the clinical trials for the management of HIT, intravenous dosing regimens ranged from 0.1 to $0.4 \mathrm{mg} \mathrm{kg}{ }^{-1} \mathrm{~h}^{-1}$ with or without a bolus for 11 to 14 days. ${ }^{5,56}$ Bleeding rates were higher than those of controls, and transfusions were required in a significant number of patients. Lepirudin has been approved for the treatment of HIT thrombosis by the health authorities of the United States, Canada, and Europe. In a study of 25 patients with 36 interventions, lepirudin was shown to be an effective anticoagulant in HIT patients undergoing PCI procedures. ${ }^{73}$

Exposure to lepirudin results in antibody formation in about half of the treated patients. ${ }^{74-76}$ These antibodies alter the pharmacokinetics of lepirudin necessitating careful monitoring to avoid bleeding complications. Reexposure to lepirudin has been linked to at least nine reported cases of severe anaphylaxis with at least five fatal outcomes. ${ }^{77}$

\section{BIVALIRUDIN}

Bivalirudin is a reversible inhibitor that is largely renally excreted. Although not approved by the FDA, it has been used to anticoagulate patients with HIT thrombosis. Bivalirudin is, however, approved for use in PCI in non-HIT patients. ${ }^{78}$

Perhaps the greatest obstacle to overcome in the management of patients with HIT is anticoagulation during surgical coronary revascularization or heart valve replacement surgery. For patients with active HIT, anticoagulation with bivalirudin was shown to be feasible in both on-pump (cardiopulmonary bypass; CPB) and off-pump (OPCAB) cardiac surgery. ${ }^{79,80}$ However, the use of any DTI in cardiac surgery is associated with inherent risks. There is no antidote for the DTIs. Dosing and monitoring guidelines have not been fully established, bleeding can be excessive, and monitoring the high drug levels is an unresolved issue. Complete efficacy against blood clotting is also a concern.

Until bivalirudin (or another anticoagulant) is FDA approved for use in cardiac surgery, heparin remains the best option. However, subsequent use of heparin after resolution of HIT can be hazardous particularly within the first 3 months. A brief exposure to heparin can be considered under compelling circumstances for patients with a history of HIT who have HIT antibodies that are not detectable by a functional platelet assay. ${ }^{51}$ In this circumstance, standard heparin protocols, restricted to the surgery itself, can be employed with a DTI or a vitamin $\mathrm{K}$ antagonist for postoperative care. $^{51,81}$

\section{DANAPAROID}

Danaparoid has been used to successfully treat HIT patients over the past 10 years. ${ }^{82-84}$ At present it is only available outside the United States. Because danaparoid has a low bleeding risk, routine monitoring is not required, except in patients with excessively low or high body weight or renal failure. ${ }^{85}$ It has a sustained effect and can be given either intravenously or subcutaneously. There is a small potential for clinically relevant crossreactivity of danaparoid with HIT antibodies in patients, so platelet counts should be monitored during the initial phase of treatment. ${ }^{86,87}$

A potential advantage of danaparoid over the DTIs is that it has multiple sites of action, including 
an anti-inflammatory effect. Thus in addition to inhibition of the coagulation system, danaparoid may be able to affect other aspects of the pathophysiology of HIT.

\section{Long-Term Anticoagulation}

For long-term anticoagulation of HIT patients, vitamin $\mathrm{K}$ antagonists (VKAs) are used, but specific dosing guidelines need to be followed to avoid thrombotic complications. ${ }^{30,51,88}$ VKA treatment can be initiated when the patient is out of the acute phase of HIT (platelet count on the rise and $>100 \times 10^{9} / \mathrm{L}$ ). It should be started at a low dose (a loading dose should not be used) while the patient is fully anticoagulated with a DTI. The DTI can be tapered off when the international normalized ratio (INR) is therapeutic and stable. VKA treatment should continue until platelet counts recover to a stable plateau or longer if clinically warranted.

DTIs prolong the prothrombin time (PT)/ INR. ${ }^{57,89-92}$ INRs $>5$ commonly occur with argatroban-warfarin cotherapy, but this does not correspond with an effect on coagulation factor levels and bleeding is not enhanced. ${ }^{58,91}$ There is a predictable linear effect of argatroban doses up to $2 \mu \mathrm{g} \mathrm{kg}{ }^{-1} \min ^{-1}$ on INRs during warfarin cotherapy, which allows for reliable prediction of the level of oral anticoagulation. ${ }^{90}$ To transition from lepirudin to VKA, the dose of lepirudin is first reduced to an aPTT ratio just above 1.5. Lepirudin is continued for 4 to 5 days with the VKA, then discontinued when the INR is therapeutic. ${ }^{55,56}$

\section{Future Anticoagulant Options}

The field of antithrombotic drug development is growing with new agents at various stages of approval. As these drugs enter the clinical trial phase, questions often are asked if the new agent can be used as an alternative anticoagulant for the management of HIT thrombosis.

\section{FACTOR XA INHIBITORS}

Factor Xa inhibitors (XaIs) like the DTIs are structurally different from heparin and could theoretically be used to anticoagulant HIT patients.

The synthetic derivative of heparin, fondaparinux, has not been studied nor approved for use in patients with HIT. However, there are several reports of successful use in this patient population. ${ }^{93-95}$ Yet there is one report of HIT-induced thrombosis associated with fondaparinux treatment. ${ }^{96}$ Because of this and other evidence of HIT-type antibodies being generated with fondaparinux treatment, ${ }^{97}$ this drug should be used with caution in patients with HIT until more is known.

Idraparinux, a sister molecule of fondaparinux with an extended half-life such that only once-weekly injections are needed, is in clinical trial. This agent may be considered for use in HIT; however, no reports have been published. Other structurally modified derivatives of fondaparinux, such as non-PF4 binding agents, which are in development, may be of interest.

Other XaIs are direct-acting small molecules that are orally administered. These include rivaroxaban and apixaban, which are currently in clinical trial. The oral DTI dabigatran can also be considered. These agents should have no cross-reactivity with HIT antibodies, nor should they generate HIT antibodies. If they are found to be useful for the clinical management of HIT, an advantage would be their application for both acute and long-term treatment.

\section{OTHER CONSIDERATIONS}

Although DTIs have made a huge advance in the clinical management of patients with HIT thrombosis, there remains an unacceptable rate of morbidity and mortality in this patient population. If one considers the pathophysiology of HIT, it seems obvious that inhibition of thrombin, though important, cannot provide complete antithrombotic management of HIT thrombosis. HIT is associated not only with a hypercoagulable state but also with platelet activation, vascular endothelial dysfunction, and inflammation (leukocyte activation, cytokine upregulation).

Limited studies suggest that a combination of an inhibitor of thrombin/thrombin generation plus an antiplatelet drug may be of interest. ${ }^{98}$ Aspirin, however, does not block HIT-induced platelet activation. ${ }^{99}$ This combined therapy targets the principle mechanisms related to the pathology of HIT: platelet activation and thrombin generation. 16,100

The XaIs may face a similar limitation as the DTIs because they, too, only target the inhibition of one coagulation factor. It is unclear if they will have the needed potency against the strong hypercoagulable state associated with HIT and the high risk of thrombosis. HIT is a multipathologic condition that may be best treated by multiple targeted therapy. Combinations of a thrombin inhibitor and a factor $\mathrm{Xa}$ inhibitor have been suggested.

Several other types of antithrombotic drugs are under consideration. Recombinant thrombomodulin is under development for use in disseminated intravascular coagulation (DIC)-associated thrombosis. It has prolonged antithrombotic activity and can be administered subcutaneously. Defibrotide is a single-stranded deoxyribonucleic acid derivative used to treat venous occlusive disease. It has multiple modes of action as an antithrombotic agent. It can be administered either by intravenous or oral routes. Sulodexide is a heparinoid complex under development for diabetic nephropathy. This drug also has multiple modes of action. It can be taken by the oral route. Although these drugs have not been studied for efficacy or safety in patients with HIT, they have the potential to be useful. 
Certain patients develop ischemic limbs or organs in which the thrombus is not alleviated with DTI anticoagulant therapy. Adjunct treatment options include thrombolytic agents and surgical removal of life- or limb-threatening thrombi. Plasmapheresis has been used to hasten reduction of antibody load in severely ill patients or in those who require cardiac surgery.

\section{CONCLUSION}

HIT is an immune response associated with platelet activation, inflammation, and an extreme hypercoagulable state resulting in thrombocytopenia and a high rate of thromboembolic complications.

Current management practice for patients with HIT is high clinical awareness, early diagnosis, and early treatment. However, the diagnosis of HIT is complicated because patients who present often do not fit the prescribed textbook definition.

The diagnosis of HIT is based on a comprehensive interpretation of clinical and laboratory information beginning with careful monitoring for thrombocytopenia and thrombosis during and for at least several days after heparin treatment of any dose and duration.

The available laboratory tests for HIT vary in their sensitivity and specificity. Each type of test provides unique information. The ELISA tests merely provide evidence of the presence of HIT antibodies that may or may not be related to clinical symptoms. Platelet function tests detect HIT antibodies that cause platelet activation and have a better correlation with patients with HIT-associated thrombocytopenia and thrombosis, but because of poorer sensitivity, a negative test result cannot exclude HIT. Appropriate use and knowledgeable interpretation of the test results are therefore very important.

It is useful to perform a combination of tests and to repeat testing over a period of several days. Initial therapeutic decisions should not be dependent upon a positive laboratory test but should be based on clinical findings (i.e., thrombocytopenia and/or new thromboembolic events). Laboratory tests should not be used to guide initial therapeutic decisions but rather to confirm a clinical diagnosis of HIT to guide future therapy.

UFH and LMWH must be stopped when the diagnosis of HIT is suspected. Because of the strong hypercoagulable state and high risk of thrombosis associated with HIT, it is recommended that all HIT patients be treated with an alternate anticoagulant. Currently, parenteral use of a DTI (such as argatroban or lepirudin) or danaparoid is recommended for the anticoagulant management of patients with HIT. Differences between drugs need to be considered when making a clinical treatment decision. Long-term management of these patients is based on the use of oral anticoagulant warfarin, but specific dosing guidelines must be followed. In the future, oral thrombin and factor $\mathrm{Xa}$ inhibitors may prove to be useful for the management of HIT.

Argatroban has been approved for use in invasive cardiology procedures in HIT patients. HIT patients who require coronary revascularization and special patient populations including pediatric patients, pregnant women, and stroke patients continue to represent a challenge.

The diagnosis and treatment of HIT is complex but needs to be considered in the clinical management of patients exposed to heparin due to its serious outcomes. Research and clinical studies will continue to address the unresolved issues and unmet clinical needs associated with HIT.

\section{REFERENCES}

1. Kelton JG, Sheridan D, Santos A, et al. Heparin-induced thrombocytopenia: laboratory studies. Blood 1988;72:925930

2. Amiral J, Bridey F, Dreyfus M, et al. Platelet factor 4 complexed to heparin is the target for antibodies generated in heparin-induced thrombocytopenia. Thromb Haemost 1992;68:95-96

3. Greinacher A, Potzsch B, Amiral J, Dummel V, Eichner A, Mueller-Eckhardt C. Heparin-associated thrombocytopenia: isolation of the antibody and characterization of a multimolecular PF4-heparin complex as the major antigen. Thromb Haemost 1994;71:247-251

4. Maccarana M, Lindahl U. Model of interaction between platelet factor 4 and heparin. Glycobiology 1993;3:271277

5. Mayo KH, Ilyina E, Roongta V, et al. Heparin binding to platelet factor-4. An NMR and site-directed mutagenesis study: arginine residues are crucial for binding. Biochem J 1995;312:357-365

6. Newman PM, Chong BH. Heparin-induced thrombocytopenia: new evidence for the dynamic binding of purified anti-PF4-heparin antibodies to platelets and the resultant platelet activation. Blood 2000;96:182-187

7. Suh JS, Aster RH, Visentin GP. Antibodies from patients with heparin-induced thrombocytopenia/thrombosis recognize different epitopes on heparin:platelet factor 4. Blood 1998;91:916-922

8. Visentin GP. Heparin-induced thrombocytopenia: molecular pathogenesis. Thromb Haemost 1999;82:448-456

9. Rauova L, Poncz M, McKenzie SE, et al. Ultralarge complexes of PF4 and heparin are central to the pathogenesis of heparin-induced thrombocytopenia. Blood 2005;105:131138

10. Visentin GP, Ford SE, Scott PJ, Aster RH. Antibodies from patients with heparin-induced thrombocytopenia/ thrombosis are specific for platelet factor 4 complexed with heparin or bound to endothelial cells. J Clin Invest 1994; 93:81-88

11. Newman PM, Chong WJ. Further characterization of antibody and antigen in heparin-induced thrombocytopenia. Br J Haematol 1999;107:303-309

12. Amiral J, Marfaing-Koka M, Wolf M, et al. Presence of autoantibodies to interleukin-8 or neutrophil-activating 
peptide-2 in patients with heparin-associated thrombocytopenia. Blood 1996;88:410-416

13. Regnault V, deMaistre E, Carteaux J, Gruel Y, Nguyen P. Platelet activation induced by human antibodies to interleukin-8. Blood 2003;101:1419-1421

14. Walenga JM, Prechel MM, Jeske WP, Bakhos M. Unfractionated heparin compared with low-molecularweight heparin as related to heparin-induced thrombocytopenia. Curr Opin Pulm Med 2005;11:385-391

15. Fareed J, Bick RL, Rao G, et al. The immunogenic potential of generic versions of low-molecular weight heparins may not be the same as the branded products. Clin Appl Thromb Hemost 2008;14:5-7

16. Jeske WP, Walenga JM, Szatkowski E, et al. Effect of glycoprotein IIb/IIIa antagonists on the HIT serum induced activation of platelets. Thromb Res 1997;88:271-281

17. Warkentin TE, Hayward CP, Boshkov LK, et al. Sera from patients with heparin-induced thrombocytopenia generate platelet-derived microparticles with procoagulant activity: an explanation for the thrombotic complications of heparininduced thrombocytopenia. Blood 1994;84:3691-3699

18. Walenga JM, Jeske WP, Prechel MM, Bakhos M. Newer insights on the mechanism of heparin-induced thrombocytopenia. Semin Thromb Hemost 2004;30(Suppl 1):5767

19. Jeske WP, Vasaiwala S, Schlenker R, Wallis E, Walenga JM. Leukocyte activation in heparin-induced thrombocytopenia. Blood 2000;96:29b

20. Pouplard C, Iochmann S, Renard B, et al. Induction of monocyte tissue factor expression by antibodies to heparinplatelet factor 4 complexes developed in heparin-induced thrombocytopenia. Blood 2001;97:3300-3302

21. Herbert JM, Savi P, Jeske WP, Walenga JM. Effect of SR121566A, a potent GP IIb-IIIa antagonist, on the HIT serum/heparin-induced platelet mediated activation of human endothelial cells. Thromb Haemost 1998;80:326331

22. Walenga JM, Michal K, Hoppensteadt D, Wood JJ, Bick RL, Robinson JA. Vascular damage correlates between heparin-induced thrombocytopenia and the antiphospholipid syndrome. Clin Appl Thromb Hemost 1999;5(Suppl 1): S76-S84

23. Fareed J, Walenga JM, Hoppensteadt DA, et al. Selectins in the HIT syndrome: pathophysiologic role and therapeutic modulation. Semin Thromb Hemost 1999;25(Suppl 1):3742

24. Blank M, Shoenfeld Y, Tavor S, et al. Anti-platelet factor 4/ heparin antibodies from patients with heparin-induced thrombocytopenia provoke direct activation of microvascular endothelial cells. Int Immunol 2002;14:121-129

25. Matsuo T, Tomaru T, Kario K, Hirokawa T. Incidence of heparin-PF4 complex antibody formation and heparininduced thrombocytopenia in acute coronary syndrome. Thromb Res 2005;115:475-481

26. Opatrny L, Warner MN. Risk of thrombosis in patients with malignancy and heparin-induced thrombocytopenia. Am J Hematol 2004;76:240-244

27. Reininger CB, Greinacher A, Graf J, Lassila R, Steckmeier B, Schwieberer L. Platelets of patients with peripheral arterial disease are hypersensitive to heparin. Thromb Res 1996;81:641-649

28. Warkentin TE, Sheppard JA, Horsewood P, Simpson PJ, Moore JC. Impact of the patient population on the risk for heparin-induced thrombocytopenia. Blood 2000;96:17031708

29. Shuster TA, Silliman WR, Coats RD, Mureebe L, Silver D. Heparin-induced thrombocytopenia: twenty-nine years later. J Vasc Surg 2003;38:1316-1322

30. Warkentin TE, Greinacher A. Heparin-induced thrombocytopenia: recognition, treatment, and prevention. Chest 2004;126:311S-337S

31. Warkentin TE, Crowther MA. When is HIT really HIT? Ann Thorac Surg 2007;83:21-23

32. Lo GK, Juhl D, Warkentin TE, Sigouin CS, Eichler P, Greinacher A. Evaluation of pretest clinical score (4 T's) for the diagnosis of heparin-induced thrombocytopenia in two clinical settings. J Thromb Haemost 2006;4:759-765

33. Pouplard C, Amiral J, Borg JY, Laporte-Simitsidis S, Delahousse B, Gruel Y. Decision analysis for use of platelet aggregation test, carbon 14-serotonin release assay, and heparin-platelet factor 4 enzyme-linked immunosorbent assay for diagnosis of heparin-induced thrombocytopenia. Am J Clin Pathol 1999;111:700-706

34. Parker RI. Measurement of heparin-dependent platelet antibodies in the diagnosis of heparin-induced thrombocytopenia: fact or fiction? Crit Care Med 2007;35:17841785

35. Walenga JM, Jeske WP, Fasanella AR, Wood JJ, Bakhos M. Laboratory tests for the diagnosis of heparin-induced thrombocytopenia. Semin Thromb Hemost 1999;25(Suppl. 1): 43-49

36. Chong BH, Burgess J, Ismail F. The clinical usefulness of the platelet aggregation test for the diagnosis of heparininduced thrombocytopenia. Thromb Haemost 1993;69:344350

37. Warkentin TE, Hayward CPM, Smith CA, Kelly PM, Kelton JG. Determinants of donor platelet variability when testing for heparin-induced thrombocytopenia. J Lab Clin Med 1992;120:371-379

38. Eichler P, Budde U, Haas S, et al. First workshop for detection of heparin-induced antibodies: validation of the heparin-induced platelet-activation test (HIPA) in comparison with a PF4/heparin ELISA. Thromb Haemost 1999; 81:625-629

39. Izban KF, Lietz JW, Hoppensteadt DA, et al. Comparison of two PF4/heparin ELISA assays for the laboratory diagnosis of heparin-induced thrombocytopenia. Semin Thromb Hemost 1999;25(Suppl 1):51-56

40. Warkentin TE, Greinacher A. Laboratory testing for heparin-induced thrombocytopenia. In: Warkentin TE, Greinacher A, eds. Heparin-Induced Thrombocytopenia. 4th ed. New York, NY: Informa Healthcare; 2007:227-260

41. Martin-Toutain I, Piette JC, Diemert MC, Faucher C, Jobic L, Ankri A. High prevalence of antibodies to platelet factor 4 heparin in patients with antiphospholipid antibodies in absence of heparin-induced thrombocytopenia. Lupus 2007;16:79-83

42. Warkentin TE, Sheppard JA, Moore JC, Moore KM, Sigouin CS, Kelton JG. Laboratory testing for the antibodies that cause heparin-induced thrombocytopenia: how much class to we need? J Lab Clin Med 2005;146:341346

43. Juhl D, Eichler P, Lubenow N, Strobel U, Wessel A, Greinacher A. Incidence and clinical significance of anti$\mathrm{PF} 4 /$ heparin antibodies of the $\operatorname{IgG}, \operatorname{IgM}$, and $\operatorname{IgA}$ class in 755 consecutive patients samples referred for diagnostic 
testing for heparin-induced thrombocytopenia. Eur J Haematol 2006;76:420-426

44. Amiral J, Bridey F, Dreyfus M, et al. Platelet factor 4 complexed to heparin is the target for antibodies generated in heparin-induced thrombocytopenia. Thromb Haemost 1992;68:95-96

45. Platelet Immunology ISTH Scientific Subcommittee. Chairs: Warkentin T, Chong B, Greinacher A, Gruel Y, Kiefel V, Kroll H. 6 July 2007. Palexpo, Geneva, Switzerland. Available at www.isth2007.com. Accessed December 6, 2007

46. Pouplard C, Gueret P, Fouassier M, Ternisien C, Trossaert M, Regina S. Prospective evaluation of the '4Ts' score and particle gel immunoassay specific to heparin/PF4 for the diagnosis of heparin-induced thrombocytopenia. J Thromb Haemost 2007;5:1373-1379

47. Warkentin TE. Platelet count monitoring and laboratory testing for heparin-induced thrombocytopenia. Arch Pathol Lab Med 2002;126:1415-1423

48. Zwicker JI, Uhl L, Huang W-Y, Shaz BH, Bauer KA. Thrombosis and ELISA optical density values in hospitalized patients with heparin-induced thrombocytopenia. J Thromb Haemost 2004;2:2133-2137

49. Refaai MA, Laposata M, VanCott EM. Clinical significance of a borderline titer in a negative ELISA test for heparin-induced thrombocytopenia. Am J Clin Pathol 2003;119:61-65

50. Smythe MA, Koerber JM, Mattson JC. An evaluation of heparin platelet factor 4 antibody testing. J Thromb Haemost 2005;3(Suppl 1):1516

51. Messmore HL, Jeske WP, Wehrmacher W, Walenga JM. Benefit-risk assessment of treatments for heparin-induced thrombocytopenia. Drug Safety 2003;26:625-641

52. Wallis DE, Workman DL, Lewis BF, Pifarré R, Moran JF. Failure of early heparin cessation as a treatment for heparin-induced thrombocytopenia. Am J Med 1999;106: 629-635

53. Lewis BE, Wallis DE, Berkowitz SD, et al. for the ARG911 Study Investigators. Argatroban anticoagulant therapy in patients with heparin-induced thrombocytopenia. Circulation 2001;103:1838-1843

54. Lewis BE, Wallis DE, Leya F, Hursting MJ, Kelton JG. for the Argatroban-915 Investigators. Argatroban anticoagulation in patients with heparin-induced thrombocytopenia. Arch Intern Med 2003;163:1849-1856

55. Greinacher A, Völpel H, Janssens U, et al. for the HIT Investigators Group. Recombinant hirudin (lepirudin) provides safe and effective anticoagulation in patients with heparin-induced thrombocytopenia: a prospective study. Circulation 1999;99:73-80

56. Greinacher A, Janssens U, Berg G, et al. for the HAT Investigataors. Lepirudin (recombinant hirudin) for parenteral anticoagulation in patients with heparin-induced thrombocytopenia. Circulation 1999;100:587-593

57. Walenga JM, Fasanella AR, Iqbal O, et al. Coagulation laboratory testing in patients treated with argatroban. Semin Thromb Hemost 1999;25(Suppl 1):61-66

58. Walenga JM, Drenth AF, Mayuga M, et al. Transition from argatroban to oral anticoagulation with phenprocoumon or acenocoumarol: effect on coagulation factor testing. Clin Appl Thromb Hemost 2008; in press

59. Hirsh J, Heddle N, Kelton JG. Treatment of heparin-induced thrombocytopenia. Arch Intern Med 2004;164:361-369
60. Warkentin TE. Management of heparin-induced thrombocytopenia: a critical comparison of lepirudin and argatroban. Thromb Res 2003;110:73-82

61. Walenga JM, Fareed D, Schultz C, Neville B, Hoppensteadt D. Argatroban, not lepirudin or bivalirudin, treatment results in the generation of nitric oxide during parenteral administration. Blood 2004;104:512a

62. Lewis BE, Walenga JM, Hursting MJ. Argatroban anticoagulation in patients with heparin-induced thrombocytopenia. Cardiovasc Rev Rep 2002;23:445-457

63. Swan SK, Hursting MJ. The pharmacokinetics and pharmacodynamics of argatroban: effects of age, gender, and hepatic or renal dysfunction. Pharmacotherapy 2000;20:318-329

64. Swan SK, St. Peter JV, Lambrecht LJ, Hursting MJ. Comparison of anticoagulant effects and safety of argatroban and heparin in healthy subjects. Pharmacotherapy 2000; 20:756-770

65. La Monte MP, Brown PM, Hursting MJ. Alternative parenteral anticoagulation with argatroban, a direct thrombin inhibitor. Expert Rev Cardiovasc Ther 2005;3:31-41

66. Matthai WH Jr, Hursting MJ, Lewis BE, Kelton JG. Argatroban anticoagulation in patients with a history of heparin-induced thrombocytopenia. Thromb Res 2005;116: 121-126

67. Walenga JM, Ahmad S, Hoppensteadt D, Iqbal O, Hursting MJ, Lewis BE. Argatroban therapy does not generate antibodies that alter its anticoagulant activity in patients with heparin-induced thrombocytopenia. Thromb Res 2002;105:401-405

68. Lewis BE, Matthai WH, Cohen M, Moses J, Hursting MJ, Leya F. Argatroban anticoagulation during percutaneous coronary intervention in patients with heparin-induced thrombocytopenia. Catheter Cardiovasc Interv 2002;57: $177-184$

69. Jang IK, Hurstings MJ. When heparins promote thrombosis: review of heparin-induced thrombocytopenia. Circulation 2005;111:2671-2683

70. Cetta F, Graham LC, Wrona LL, Arruda MJ, Walenga JM. Argatroban use during pediatric interventional cardiac catheterization. Catheter Cardiovasc Interv 2004;61:147149

71. Jang IK, Lewis BE, Matthai WH, Kleiman NS. Argatroban anticoagulation in conjunction with glycoprotein IIb/IIIa inhibition in patients undergoing percutaneous coronary intervention: an open-label, nonrandomized pilot study. J Thromb Thrombolysis 2004;18:31-37

72. LaMonte MP, Brown PM, Hursting MJ. Stroke in patients with heparin-induced thrombocytopenia and the effect of argatroban therapy. Crit Care Med 2004;32:976-980

73. Cochran K, DeMartini TJ, Lewis BE, et al. Use of lepirudin during percutaneous vascular interventions in patients with heparin-induced thrombocytopenia. J Invasive Cardiol 2003; 15:617-621

74. Eichler P, Friesen HJ, Lubenow N, Jaeger B, Greinacher A. Anti-hirudin antibodies in patients with heparininduced thrombocytopenia treated with lepirudin: incidence, effects on aPTT, and clinical relevance. Blood 2000; 96:2373-2378

75. Greinacher A, Eichler P, Albrecht D, Strobel U, Potzsch B, Eriksson BI. Anti-hirudin antibodies following low-dose subcutaneous treatment with desirudin for thrombosis prophylaxis after hip-replacement surgery: incidence and clinical relevance. Blood 2003;101:2617-2619 
76. Fischer KG, Liebe V, Hudek R, et al. Anti-hirudin antibodies alter pharmacokinetics and pharmacodynamics of recombinant hirudin. Thromb Haemost 2003;89:973982

77. Greinacher A, Lubenow N, Eichler P. Anaphylactic and anaphylactoid reactions associated with lepirudin in patients with heparin-induced thrombocytopenia (HIT). Circulation 2003;108:2062-2065

78. Mahaffey KW, Lewis BE, Wildermann NMet alfor the ATBAT Investigators. The anticoagulant therapy with bivalirudin to assist in the performance of PCI in patients with heparin-induced thrombocytopenia (ATBAT) study. J Invasive Cardiol 2003;15:611-616

79. Merry AF, Raudkivi PJ, Middleton NG, et al. Bivalirudin versus heparin and protamine in off-pump coronary artery bypass surgery. Ann Thorac Surg 2004;77:925-931

80. Koster A, Spiess B, Chew DP, et al. Effectiveness of bivalirudin as a replacement for heparin during cardiopulmonary bypass in patients undergoing coronary artery bypass grafting. Am J Cardiol 2004;93:356-359

81. Pötzsch B, Klovekorn WP. Use of heparin during cardiopulmonary bypass in patients with a history of heparin-induced thrombocytopenia. N Engl J Med 2000; 343:515-516

82. Magnani HN, Gallus A. Heparin-induced thrombocytopenia (HIT). A report of 1,478 clinical outcomes of patients treated with danaparoid (Orgaran) from 1982 to mid-2004. Thromb Haemost 2006;95:967-981

83. Lindhoff-Last E, Kreutzenbeck H-J, Magnani HN. Treatment of 51 pregnancies with danaparoid because of heparin intolerance. Thromb Haemost 2005;93:63-69

84. Chong BH, Gallus AS, Cade JF, Magnani H, Manoharan A, Oldmeadow Mfor the Australian HIT Study Group. Prospective randomized open-label comparison of danaparoid with dextran 70 in the treatment of heparin-induced thrombocytopenia with thrombosis. Thromb Haemost 2001; 86:1170-1175

85. Farner B, Eichler P, Kroll H, Greinacher A. A comparison of danaparoid and lepirudin in heparin-induced thrombocytopenia. Thromb Haemost 2001;85:950-957

86. Walenga JM, Koza MJ, Lewis BE, Pifarré R. Relative heparin-induced thrombocytopenic potential of low molecular weight heparins and new antithrombotic agents. Clin Appl Thromb Hemost 1996;2(Suppl 1):S21-S27

87. Elalamy I, Lecrubier C, Horellou MH, Conard J, Samama MM. Heparin-induced thrombocytopenia: laboratory management. Ann Med 2000;32(Suppl 1):60-67

88. Wallis DE, Quintos R, Wehrmacher W, Messmore HL. Safety of warfarin anticoagulation in patients with heparin-induced thrombocytopenia. Chest 1999;116:13331338

89. Gosselin RC, Dager WE, King JH, et al. Effect of direct thrombin inhibitors, bivalirudin, lepirudin, and argatroban, on prothrombin time and INR values. Am J Clin Pathol 2004; 121:593-599

90. Sheth SB, DiCicco RA, Hursting MJ, Montague T, Jorkasky DK. Interpreting the international normalized ratio (INR) in individuals receiving argatroban and warfarin. Thromb Haemost 2001;85:435-440

91. Harder S, Graff J, Klinkhardt U, et al. Transition from argatroban to oral anticoagulation with phenprocoumon or acenocoumarol: effects on PT, aPTT, and ecarin clotting time. Thromb Haemost 2004;91:1137-1145

92. Hursting MJ, Lewis BE, Macfarlane DE. Transitioning from argatroban to warfarin therapy in patients with heparin-induced thrombocytopenia. Clin Appl Thromb Hemost 2005;11:279-287

93. Parody R, Oliver A, Souto JC, Fontcuberta J. Fondaparinux (Arixtra) as an alternative anti-thrombotic prophylaxis when there is hypersensitivity to low molecular weight and unfractionated heparins. Haematologica 2003;88: ECR32

94. D'Amico EA, Villaca PR, Gualandro SF, Bassitt RP, Chamone DA. Successful use of Arixtra in a patient with paroxysmal nocturnal hemoglobinuria, Budd-Chiari syndrome and heparin-induced thrombocytopenia. J Thromb Haemost 2003;1:2452-2453

95. Bradner J, Hallisey RK, Kuter DJ. Fondaparinux in the treatment of heparin-induced thrombocytopenia. Blood 2004;104: abstract 1775

96. Warkentin TE, Maurer BT, Aster RH. Heparin-induced thrombocytopenia associated with fondaparinux. N Engl J Med 2007;356:2653-2655

97. Warkentin TE, Cook RJ, Marder VJ, et al. Anti-platelet factor 4/heparin antibodies in orthopedic surgery patients receiving antithrombotic prophylaxis with fondaparinux or enoxaparin. Blood 2005;106:3791-3796

98. Walenga JM, Jeske WP, Wallis DE, et al. Clinical experience with combined treatment of thrombin inhibitors and GPIIb/IIIa inhibitors in patients with HIT. Semin Thromb Hemost 1999;25(Suppl 1):77-81

99. Walenga JM, Lewis BE, Jeske WP, et al. Combined thrombin and platelet inhibition treatment for HIT patients. Haemostaseologie 1999;19:128-133

100. Haas S, Walenga JM, Jeske WP, Fareed J. Heparin-induced thrombocytopenia: the role of platelet activation and therapeutic implications. Semin Thromb Hemost 1999; 25(Suppl 1):67-75 